\title{
MicroRNA-200a suppresses epithelial-to-mesenchymal transition in rat hepatic stellate cells via GLI family zinc finger 2
}

\author{
FUJUN YU ${ }^{1}$, YIHU ZHENG ${ }^{2}$, WEILONG HONG ${ }^{3}$, BICHENG CHEN $^{3}$, PEIHONG DONG $^{1}$ and JIANJIAN ZHENG ${ }^{3}$ \\ Departments of ${ }^{1}$ Infectious Diseases and ${ }^{2}$ General Surgery; ${ }^{3}$ Key Laboratory of Surgery, \\ The First Affiliated Hospital of Wenzhou Medical University, Wenzhou, Zhejiang 325000, P.R. China
}

Received December 31, 2014; Accepted September 25, 2015

DOI: $10.3892 / \mathrm{mmr} .2015 .4452$

\begin{abstract}
Hepatic stellate cells (HSCs) have an important role in liver fibrosis. Epithelial-to-mesenchymal transition (EMT), which is promoted by the Hedgehog $(\mathrm{Hh})$ signaling pathway, is involved in the activation of HSCs. MicroRNAs (miRNAs/miRs) have been reported to be involved in the progression of liver fibrosis. A previous study indicated that the activation of HSCs was suppressed by miR-200a via targeting transforming growth factor- $\beta 2$ and $\beta$-catenin. However, whether miR-200a is able to regulate the EMT in HSCs has remained elusive. The present study revealed that miR-200a was decreased in vitro and in vivo during liver fibrosis. Furthermore, miR-200a overexpression resulted in the inhibition of proliferation, $\alpha$-SMA expression and extracellular matrix production of activated HSCs. Of note, miR-200a overexpression reduced myofibroblastic markers, including $\alpha$-SMA, type I collagen and desmin, and increased the epithelial cell marker E-cadherin. These results were further confirmed by immunofluorescence staining. Further study showed that the expression of genes associated with $\mathrm{Hh}$ signaling, including Hhip, Shh and Gli1, were not affected by miR-200a. However, Gli2, a downstream signaling protein of the Hh pathway, was inhibited by miR-200a and confirmed as a target of miR-200a using a dual luciferase reporter assay. In addition, the inhibition of the Hh pathway by miR-200a resulted in an increase of BMP-7 and Id 2 as well as a reduction of Snail and S100A4. Collectively, the results of the present study demonstrated that miR-200a suppressed the EMT process in HSCs, at least in part, via Gli2.
\end{abstract}

Correspondence to: Mr. Jianjian Zheng, Key Laboratory of Surgery, The First Affiliated Hospital of Wenzhou Medical University, 2 Fuxue Lane, Wenzhou, Zhejiang 325000, P.R. China E-mail:120378196@qq.com

Dr Peihong Dong, Department of Infectious Diseases, The First Affiliated Hospital of Wenzhou Medical University, 2 Fuxue Lane, Wenzhou, Zhejiang 325000, P.R. China

E-mail: dongpeihong111@163.com

Key words: microRNA-200a, epithelial-to-mesenchymal transition, hepatic stellate cells, hedgehog pathway, GLI family zinc finger 2

\section{Introduction}

Liver fibrosis, characterized by excess production and deposition of extracellular matrix (ECM) along with loss of liver function and disruption of liver structure, is a wound-healing response to chronic liver injury $(1,2)$. It is well known that hepatic stellate cells (HSCs) have an important role in liver fibrosis. The activation and proliferation of resident hepatic stellate cells (HSCs) has been considered as a central event in the progression of liver fibrosis. During fibrosis progression, quiescent (Q)-HSCs become activated and transdifferentiate into myofibroblastic HSCs (MF-HSCs) that express smooth-muscle $\alpha$-actin ( $\alpha$-SMA) as a marker (3). Activation of HSC, the major cell type promoting synthesis and deposition of ECM proteins, results in an imbalance between ECM protein generation and their degradation in the liver (4). Therefore, a possible therapeutic strategy for treating liver fibrosis is to inhibit the activation of HSCs.

Epithelial-to-mesenchymal transition (EMT) is the process by which epithelial cells gradually lose their epithelial signatures while acquiring the characteristics of mesenchymal cells (5). A growing body of evidence implied that EMT has an important role in liver fibrosis (6,7). In particular, it has been confirmed that myofibroblasts can be supplemented from hepatocytes by EMT during hepatic fibrosis (8). EMT has emerged as a promising therapeutic target for the attenuation of liver fibrosis. Hedgehog (Hh) signaling has been identified to be involved in EMT. Choi et al (9) demonstrated that EMT is regulated by Hh signaling during myofibroblastic transformation of rat hepatic cells in culture and cirrhosis. Omenetti et al (7) found that EMT responses to bile duct ligation were enhanced in patched-deficient mice, which display excessive activation of the Hh pathway. A previous study showed that leptin, a pro-EMT factor, activates Hh signaling to alter the expression of genes which control cell fate and have important implications in liver fibrosis (10). These findings suggested that Hh signaling contributes to EMT.

MicroRNAs (miRNAs) are endogenous small (18-22 nt) non-coding RNAs that regulate the expression of proteins involved in diverse cellular and developmental processes, including differentiation, apoptosis and oncogenesis (11). miRNAs specifically bind to the 3'-untranslated region (3'-UTR) of their respective target mRNAs to decrease their stability and translation to regulate protein expression (12). 
During liver fibrosis, miRNAs contribute to the activation status of HSCs and function as HSC regulators in liver fibrosis. Sun et al (13) reported that miR-200a overexpression suppressed HSC activation and proliferation by targeting TGF- $\beta 2$ and $\beta$-catenin. Given the complexity of the EMT, it is likely that miRNAs regulate most genes involved in the EMT. Previously, it has been reported that the miR-200 family regulates transforming growth factor (TGF)- $\beta 1$-induced renal tubular EMT through the Smad pathway by targeting the expression of zinc finger E-box binding homeobox 1 (ZEB1) and ZEB2 (14). However, it has remained elusive whether miR-200a regulates EMT in HSCs.

The present study assessed the effects of miR-200a on the proliferation of activated HSCs; furthermore, western blot analysis, reverse-transcription quantitative polymerase chain reaction (RT-qPCR) and immunofluorescence were used to assess the expression of epithelial marker E-cadherin and myofibroblastic markers $\alpha$-SMA, type I collagen and desmin in order to investigate the effects of miR-200a on the EMT of cultured HSCs. In addition, the effects of miR-200a on the expression of Hh pathway-associated genes, including Hh-interacting protein (Hhip), Sonic Hh (Shh) and GLI family zinc finger 1 (Gli1) and Gli2, as well as genes associated with the EMT, including bone morphogenetic protein-7 (BMP-7), inhibitor of DNA binding 2 (Id2), Snail family zinc finger 1 (Snai1) and S100 calcium-binding protein A4 (S100A4) were assessed. A luciferase reporter assay was then employed to investigate whether Gli2 was a direct target of miR-200a. The present study suggested that miR-200a suppressed EMT in rat HSCs, at least in part, via Gli2.

\section{Materials and methods}

Materials. $\mathrm{CCl}_{4}$ was obtained from Sigma-Aldrich (St. Louis, MO, USA) and antibodies, including rabbit polyclonal anti-type I collagen (cat. no. ab34710), anti-desmin (cat.no. ab15200) and anti-S100A4 (cat.no. ab27957), and mouse monoclonal anti-E-cadherin (cat. no. ab76055), anti- $\alpha$-SMA (cat. no. ab7817), and anti- $\beta$-actin (cat. no. ab6276) were obtained from Abcam (Cambridge, MA, USA). Antibodies including goat polyclonal anti-BMP-7 (cat. no. sc-9305), anti-Id2 (cat. no. sc-26328), and anti-Hhip (cat. no. sc-9406), and rabbit polyclonal anti-Snail (cat. no. sc-28199), anti-Shh (cat. no. sc-9024), anti-Gli1 (cat. no. sc-20687) and anti-Gli2 (cat. no. sc-28674) were purchased from Santa Cruz Biotechnology (Dallas, TX, USA).

Isolation and culture of rat HSCs. Adult male Sprague-Dawley rats (body weight, 400-500 g) were used for HSC isolation as described previously (15). The liver tissues were digested with collagenase IV $(0.5 \mathrm{~g} / \mathrm{l})$ and deoxyribonuclease I (0.03 g/l) prior to fractionation on a discontinuous gradient of iodixanol. HSCs were harvested from the $11.5 \%$ medium interface, washed and seeded in tissue culture plates. Cells were cultured in Dulbecco's modified Eagle's medium (DMEM; Gibco, Thermo Fisher Scientific, Waltham, MA, USA) with $10 \%$ fetal bovine serum (FBS; Sigma-Aldrich), $100 \mathrm{U} / \mathrm{ml}$ penicillin and $100 \mu \mathrm{g} / \mathrm{ml}$ streptomycin (Gibco; Thermo Fisher Scientific, Inc.). The harvested primary HSCs were used for subsequent experiments at day 3 after isolation. The purity of cultures
$(>98 \%)$ was confirmed by immunocytochemical staining for $\alpha$-SMA. The present study was approved by the University Animal Care and Use Committee of the Wenzhou Medical University (Wenzhou, China).

miRNA transfection. Cells were seeded in a six-well plate at a density of $1 \times 10^{6}$ cells per well. The following day, medium was replaced with Opti-Minimum Essential Medium (Invitrogen; Thermo Fisher Scientific) and cells were transiently transfected with miR-200a mimics $(60 \mathrm{nM})$ or the miR-negative control (NC; $60 \mathrm{nM}$; GenePharma, Shanghai, China) using Lipofectamine 2000 (Invitrogen) for $48 \mathrm{~h}$ in all experiments.

Rat model of $\mathrm{CCl}_{4}$-induced liver injury. Male Sprague-Dawley rats ( $\mathrm{n}=20$; weighy, 180-220 g; age, 7 weeks) were obtained from the Experimental Animal Center of the Wenzhou Medical University, and housed in an environmentally controlled room $\left(23 \pm 2^{\circ} \mathrm{C} ; 55 \pm 10 \%\right.$ humidity $)$ with a $12 \mathrm{~h}$ light/ dark cycle, and were provided ad libitum access to food and water. Liver fibrosis was generated by a 6 week treatment with $\mathrm{CCl}_{4}\left[\mathrm{CCl}_{4} /\right.$ olive oil, 1:1 (v/v) per kg body weight by intraperitoneal injection twice weekly] for six weeks as previously described (16). Twenty rats were randomly divided into two groups. Rats in group $1(n=10)$ received injections with olive oil (vehicle control) and rats in group $2(\mathrm{n}=10)$ received injections of $\mathrm{CCl}_{4}$ twice weekly. The animal experimental protocol was approved by the University Animal Care and Use Committee (Wenzhou Medical University, Wenzhou, China). Rats were sacrificed under anesthesia by intraperitoneal injection of $10 \%$ chloral hydrate (Sigma-Aldrich; $4 \mathrm{ml} / \mathrm{kg}$ body weight) after the last $\mathrm{CCl}_{4}$ injection and liver tissues were harvested for further analysis.

Immunofluorescence microscopy. Primary HSCs on day 3 following inoculation were plated on $18-\mathrm{mm}$ cover glasses in DMEM at a density of $1 \times 10^{5}$ cells/well and incubated for $24 \mathrm{~h}$. The cells were then transfected with miR-200a mimics or miR-NC for $48 \mathrm{~h}$. Following washing with phosphate-buffered saline (PBS; Wenzhou Changfeng Biotechnology Co., Ltd., Wenzhou, China) and fixing in acetic acid/ethanol (30\% ethanol and $10 \%$ acetic acid; Wenzhou Changfeng Biotechnology Co., Ltd.) for $5 \mathrm{~min}$ at $-20^{\circ} \mathrm{C}$, non-specific binding was blocked with 5\% goat serum (Sigma-Aldrich) in PBS for $1 \mathrm{~h}$ at room temperature and the cells were then incubated overnight at $4^{\circ} \mathrm{C}$ with primary antibodies against $\alpha$-SMA (1:200), type I collagen (1:100), E-cadherin (1:100) or desmin (1:100) in a humidified chamber. After washing twice in PBS, the cells were incubated for $1 \mathrm{~h}$ at room temperature with fluorescein-labeled secondary antibody (1:50 dilution; Dianova, Hamburg, Germany) in antibody dilution solution (Beyotime Institute of Biotechnology, Haimen, China) for $1 \mathrm{~h}$ at room temperature in the dark. The nuclei were stained with 4,6-diamidino-2-phenylindole (DAPI; Abcam) in the dark for $30 \mathrm{~min}$ at room temperature. The slides were washed twice with PBS, covered with DABCO (Sigma-Aldrich), and examined using confocal laser scanning microscopy (FV-1000; Olympus, Tokyo, Japan) at 488 and $568 \mathrm{~nm}$.

$R T-q P C R$. The cells were collected and total RNA was extracted from cells using the miRNeasy Mini kit (Qiagen, 
A

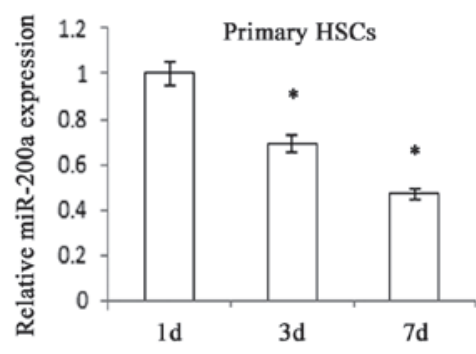

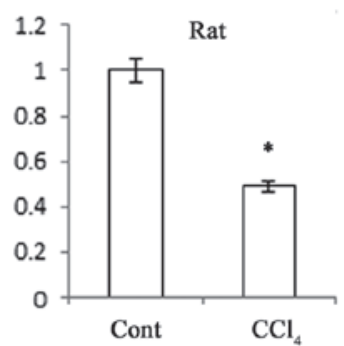

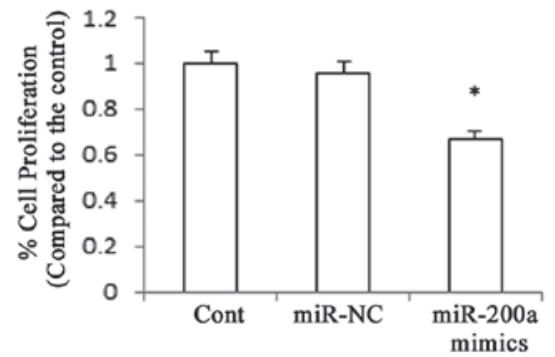

C

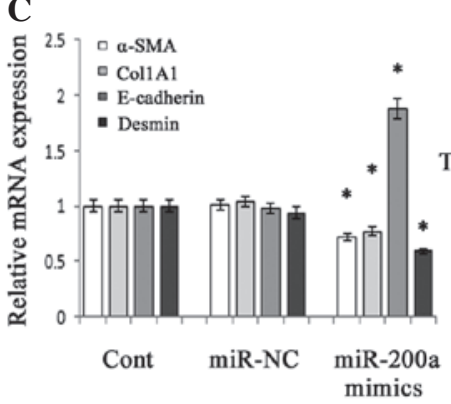

D
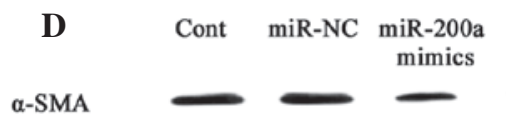

Type I collagen $-\longrightarrow$

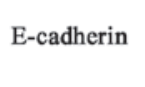

Desmin

$\beta$-actin

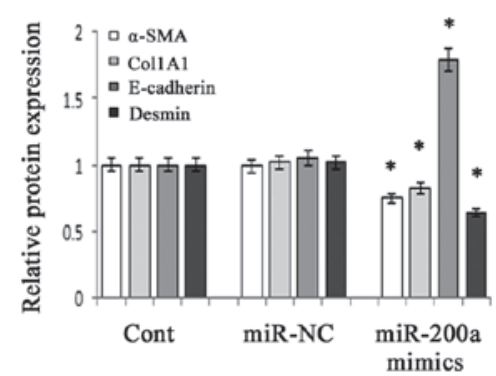

Figure 1. miR-200a is decreased during HSC activation and contributes to the suppression of activated HSCs. Primary HSCs at three days after isolation were

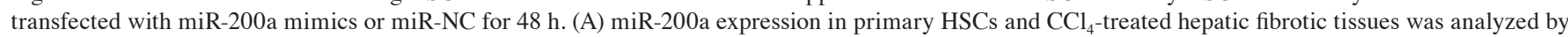
RT-qPCR. (B) The growth rate, which was analyzed using an MTT assay, was decreased by miR-200a mimics. (C) The mRNA expression of $\alpha-S M A$, Col1A1, E-cadherin and desmin in primary HSCs was analyzed by RT-qPCR. (D) The protein expression of $\alpha$-SMA, type I collagen, E-cadherin and desmin was analyzed by western blot analysis in primary HSCs. $\beta$-actin was used as an internal control. Values are expressed as the mean \pm standard deviation of three experiments. ${ }^{*} \mathrm{P}<0.05$ vs. control. miR, microRNA; HSC, hepatic stellate cell; $\alpha$-SMA, smooth-muscle $\alpha$-actin; RT-qPCR, reverse-transcription quantitative polymerase chain reaction; Cont, control; NC, negative control.

Hilden, Germany) and cDNA was synthesized according to the manufacturer's instructions (Toyobo, Osaka, Japan). Gene expression was measured by real-time PCR using cDNA, SYBR Green real-time PCR Master Mix (Toyobo), and a set of gene-specific oligonucleotide primers (Invitrogen; Thermo Fisher Scientific, Inc.): Id 2 forward, 5'-CCTCCTACG AGCAGCATGAA-3' and reverse, 5'-GGCACCAGTTCC TTGAGCTT-3'; desmin forward, 5'-ATGTCACACCCAGTC GCTTT-3' and reverse, 5'-GATGGCAGGGAAAAGGGT CA-3'; Gli1 forward, 5'-TTGCAGCCAGGAGTTCGATT-3' and reverse, 5'-GGACTTCCGACAGCCTTCAA-3'. The sequences of primers for Col1A1, $\alpha$-SMA, GAPDH, U6, E-cadherin, BMP-7, S100A4, Snai1, Hhip, Shh and Gli2 identical to those used in previous studies $(9,17)$. To detect miR-200a expression, the RT reaction was performed using the TaqMan MicroRNA assay (Applied Biosystems, Thermo Fisher Scientific) according to the manufacturer's instructions. RT-qPCR was performed in an ABI 7500 Real Time PCR system (Applied Biosystems), according to the manufacturer's protocol, with the following cycles: $95^{\circ} \mathrm{C}$ for $10 \mathrm{~min}$, followed by 40 cycles of $95^{\circ} \mathrm{C}$ for $15 \mathrm{sec}$, and $60^{\circ} \mathrm{C}$ for $60 \mathrm{sec}$. GAPDH and U6 small nuclear RNA levels were measured and used to normalize the relative abundance of mRNAs and miRNA, respectively. The $2^{-\Delta \Delta \mathrm{Ct}}$ method was used to determine the miRNA and mRNA levels as described previously (18). In addition, the specificity of RT-qPCR products was confirmed by melting curve analysis.

Protein extraction and western blot analysis. The protein concentration of samples was determined using a bicinchoninic acid protein assay kit (Beyotime Institute of Biotechnology).
Total protein (20-50 $\mu \mathrm{g})$ was subjected to $10-12 \%$ SDS-PAGE (Beyotime Institute of Biotechnology) and then transferred onto Immobilon $\mathrm{P}$ membranes (Beyotime Institute of Biotechnology). The membranes were incubated in blocking buffer [(Beyotime Institute of Biotechnology) 5\% non-fat milk powder in Tris-buffered saline with Tween 20 (TBST; $100 \mathrm{mM}$ Tris- $\mathrm{HCl} \mathrm{pH} 7.5,0.9 \% \mathrm{NaCl}, 0.1 \%$ Tween 20)] for $3 \mathrm{~h}$ at room temperature, followed by incubation overnight at $4{ }^{\circ} \mathrm{C}$ with gentle agitation with specific primary antibodies against type I collagen $(1: 1,000)$, desmin $(1: 1,000)$, S100A4 $(1: 1,000)$, E-cadherin (1:500), $\alpha$-SMA (1:500), $\beta$-actin (1:2,000), BMP-7 (1:500), Id2 (1:1,000), Hhip (1:1,000), Snai1 (1:500), Shh (1:500), Gli1 (1:500), and Gli2 (1:500). Following washing with TBST, the membranes were incubated with peroxidase-conjugated secondary antibodies (Fuzhou Maixin Biological Technology Co., Ltd., Fujian, China) for $1 \mathrm{~h}$ at room temperature. Unbound antibody was washed and removed with TBST, the antigen-antibody complex was developed by enhanced chemiluminescence using BeyoECL Plus (cat. no. P0018; Beyotime Institute of Biotechnology), and images were captured using a Gel Imager (WD-9413B; Beijing Liuyi Biotechnology Co., Ltd., Beijing, China) in a dark room and analyzed for integral absorbance (IA) of the protein bands using Quantity One 4.4 software (Bio-Rad Laboratories, Inc., Hercules, CA, USA).

Proliferation assay. Cell proliferation was determined using the MTT assay (Beyotime Institute of Biotechnology) according to the manufacturer's instructions. Briefly, the cells were seeded at a density of $5 \times 10^{3}$ cells per well in 96-well culture plates and transfected with miR-200a mimics or miR-NC. The cells were incubated with $0.5 \%$ MTT for $4 \mathrm{~h}$. 

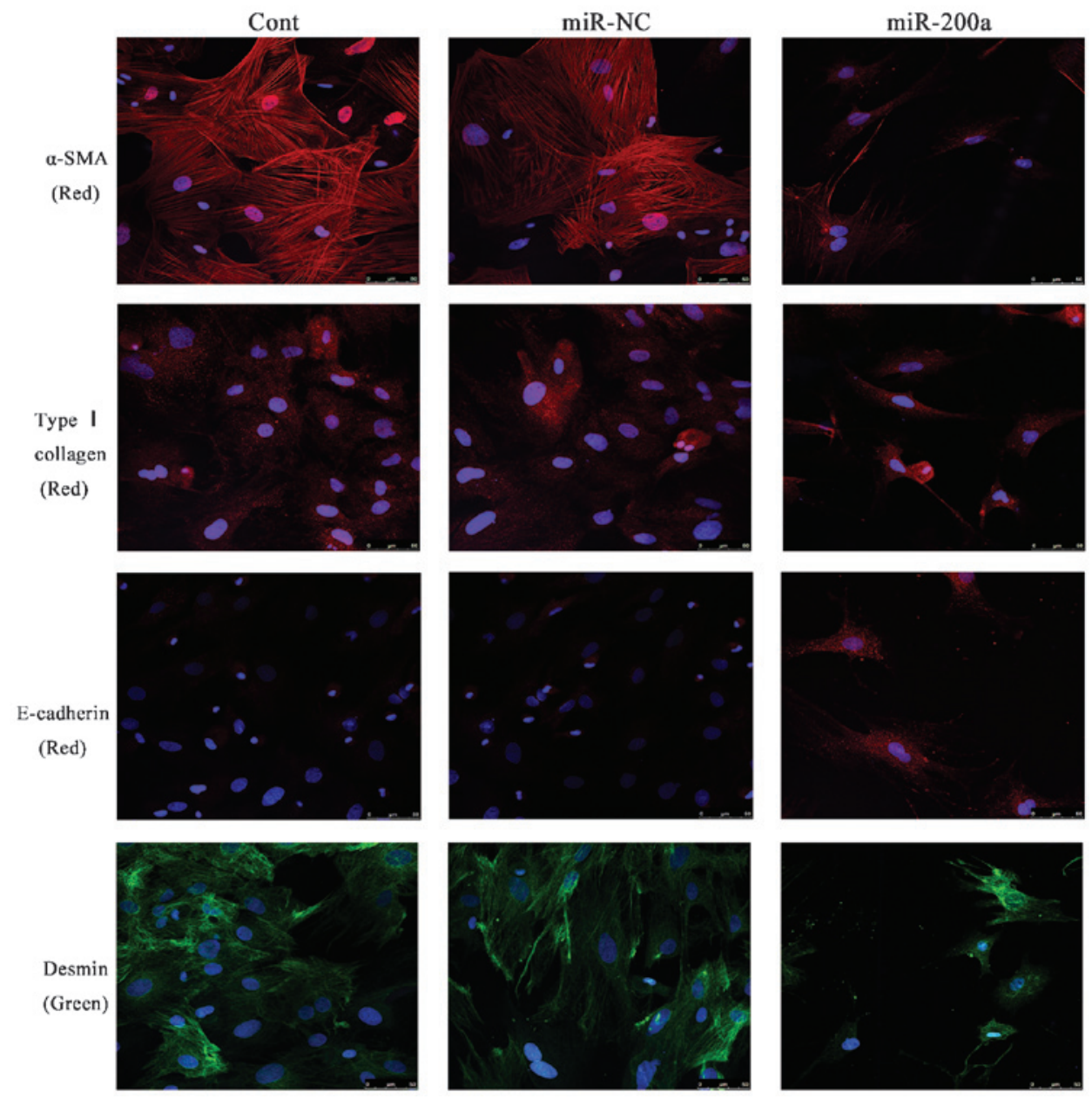

Figure 2. miR-200a contributes to the suppression of the epithelial-mesenchymal transition. Hepatic stellate cells at day 3 after isolation and were transfected with miR-200a mimics or miR-NC for $48 \mathrm{~h}$. Immunofluorescence staining for $\alpha$-SMA (red), type I collagen (red), E-cadherin (red) and desmin (green) were evaluated by confocal laser microscopy. Nuclei were stained with DAPI (blue). Scale bar, $50 \mu \mathrm{m}$. miR, microRNA; NC, negative control; Cont, control; $\alpha$-SMA, smooth-muscle $\alpha$-actin.

Following removal of the supernatant, $150 \mu \mathrm{l}$ dimethyl sulfoxide (Sigma-Aldrich) was added and plates were agitated for 5 min until the formazan crystals had dissolved. The optical density was determined using a microplate reader (Bio-Rad 550; Bio-Rad Laboratories, Inc.) at $490 \mathrm{~nm}$ wavelength. All experiments were performed in triplicate and repeated at least three times.

Luciferase reporter assay. According to a target analysis with Targetscan (http://www.targetscan.org/), sequences containing rat Gli2 3'-UTR target sequence (located at 651-657 bp), were amplified and cloned into the pMIR-Report ${ }^{\mathrm{TM}}$ Luciferase plasmid (Applied Biosystems) using mouse cDNA as template to generate pMIR-Gli2-200a vector. The primers for Gli2-3'-UTR (forward, 5'-TGCATCCATGAAGTTCGCCA-3' and reverse, 5'-GAGAGGTCAG GGGACCAGAA-3') were obtained from Invitrogen (Thermo Fisher Scientific, Inc.). The amplification conditions for Gli2-3'-UTR were the same as those demonstrated in the RT-qPCR section. pMIR-Gli2-200a-Mut was generated using a Site-Directed Mutagenesis kit (Agilent Technologies, Inc., Santa Clara, CA, USA) according to the manufacturer's instructions, using the primers containing the desired mutation (19), provided by Dr Zhou (Yuying Children's
Hospital of Wenzhou Medical University, Wenzhou, China). In addition, empty vector pMIR without the inserts was used as a negative control. pMIR-Report $\beta$-gal control plasmid was used for transfection normalization. Cells were cultured in 24-well plates and transfected with $800 \mathrm{ng}$ pMIR-200a or pMIR together with $100 \mathrm{ng}$ pMIR- $\beta$-gal and 20 pmol miR-200a precursor or miRNA negative control (miR-NC) (GenePharma). Lipofectamine 2000 (Invitrogen) was used for transfection. Forty-eight hours after transfection, luciferase and $\beta$-gal activity were measured using the Dual-Light System (Applied Biosystems).

Statistical analysis. Values are expressed as the mean \pm standard deviation from at least three independent experiments. Statistical analysis was performed using Student's t-test and $\mathrm{P}<0.05$ was considered to indicate a statistically significant difference. All statistical analyses were performed with SPSS software (version 13; SPSS, Inc., Chicago, IL, USA).

\section{Results}

MiR-200a is decreased in fibrotic livers and its upregulation suppresses the proliferation of HSCs. The present study used 
A

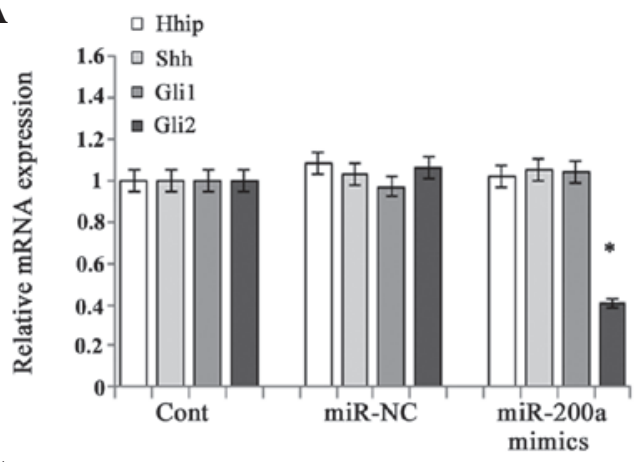

C

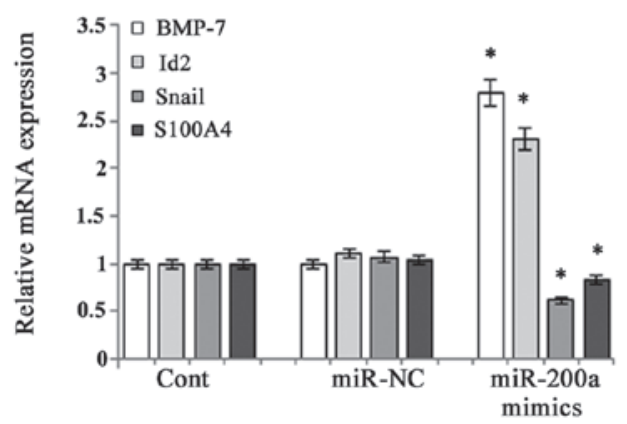

B

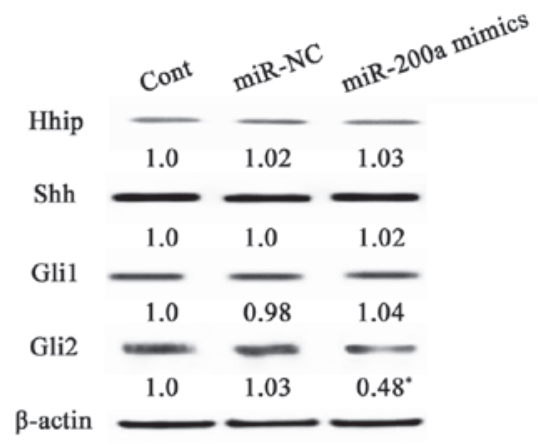

D

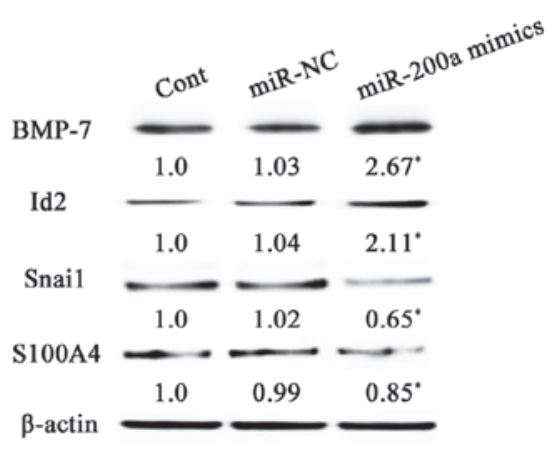

Figure 3. Effects of miR-200a on Hedgehog pathway activity. Primary HSCs on day 3 after isolation and were transfected with miR-200a mimics or miR-NC for 48 h. (A) The mRNA expression of Hhip, Shh, Gli1 and Gli2 in primary HSCs was analyzed by RT-qPCR. (B) The protein expression of Hhip, Shh, Gli1 and Gli2 in primary HSCs was assessed by western blot analysis. $\beta$-actin was used as an internal control. (C) The mRNA expression of BMP-7, Id-2, Snail and S100A4 in primary HSCs was analyzed by RT-qPCR. (D) The protein expression of BMP-7, Id2, Snail and S100A4 was assessed by western blot analysis in primary HSCs. $\beta$-actin was used as an internal control. Values are expressed as the mean \pm standard deviation of three experiments. "P<0.05 compared with the control. HSC, hepatic stellate cell; RT-qPCR, reverse-transcription quantitative polymerase chain reaction; Cont, control; NC, negative control; BMP, bone morphogenetic protein; Id2, inhibitor of DNA binding 2; S100A4, S100 calcium-binding protein A4; Shh, Sonic hedgehog; Hhip, Hedgehog-interacting protein; GL1, GLI family zinc finger 1 .

A

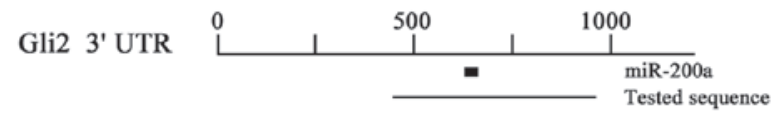

pMIR-200a

Position 651-657 of Gli2 3' UTR

Rno-miR-200a

pMIR-200a-Mut

Position 651-657 of Gli2 3' UTR Mut 5' ...UGGCCUGUUUACATCTCUC..

Rno-miR-200a

\section{5 ' ...UGgCcuguUUACAguguUC...}

3' AGCAAUGgUCUGUCACAAU

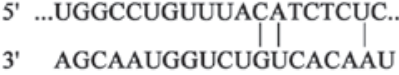

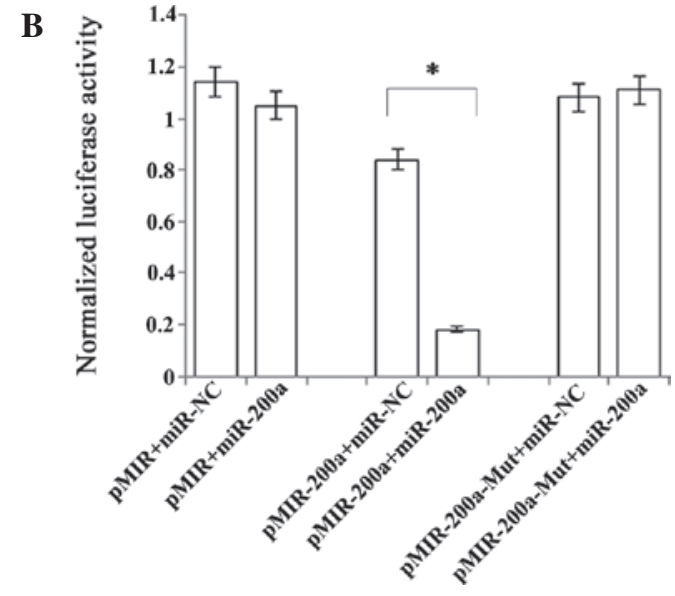

Figure 4. Interaction of miR-200a with the 3'UTR of Gli2 in primary HSCs. (A) Schematic of the miRNA binding sites in the 3'UTR of Gli2 mRNA based on Targetscan. The black box indicates miR-200a and the horizontal line (tested sequence) indicates the region that was inserted into the luciferase reporter vector. Nucleotide sequences shown illustrate the predicted consequential pairing of the target region and miR-200a. According to the pairing sites, the corresponding luciferase reporter vectors were named pMIR-200a and pMIR-200a-Mut. (B) HSCs were transfected with pMIR (empty vector), pMIR containing miR-200a targeting sequence (pMIR-200a) and pMIR with miR-200a mutated target sequence (pMIR-200a-Mut). The graph shows luciferase activity in cells transfected with pMIR-200a or pMIR-200a-Mut. It also shows co-transfection of miR-200a precursor or miR-NC. *P<0.05. UTR, untranslated region; HSC, hepatic stellate cell; miR, microRNA; Mut, mutant; GL2, GLI family zinc finger 2.

standard techniques to isolate and culture HSC from the livers of healthy rats. The expression of miR-200a was detected in primary HSCs using RT-qPCR, which revealed that miR-200a expression gradually decreased with increasing time in culture (Fig. 1A). In addition, a reduction of miR-200a expression was detected in liver tissues from $\mathrm{CCl}_{4}$-treated rats compared with that in the control animals (Fig. 1A). An MTT assay revealed that overexpression of miR-200a significantly suppressed HSC proliferation $(\mathrm{P}<0.05)$ (Fig. 1B).

miR-200a suppresses EMT in activated HSCs. Type I collagen, $\alpha$-SMA and desmin are considered to be 


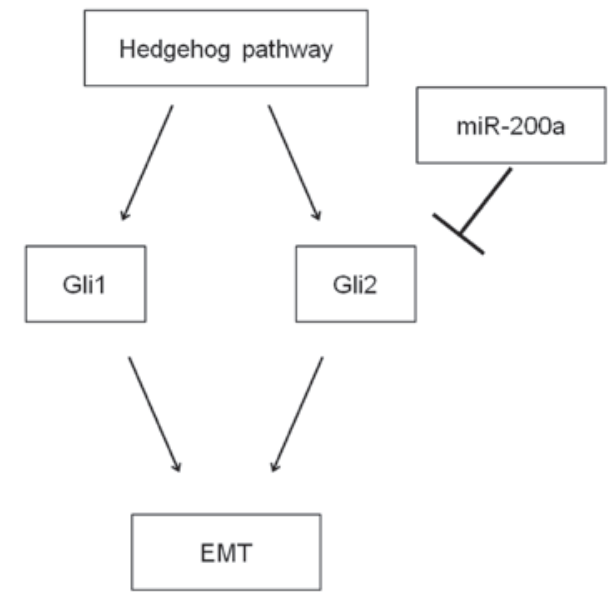

Figure 5. The EMT process was suppressed by miR-200a in rat hepatic stellate cells. Gli2, a downstream of the Hedgehog pathway, was suppressed by miR-200a, leading to the inhibition of the EMT process. EMT, epithelial-mesenchymal transition; miR, microRNA; GL1, GLI family zinc finger 1.

myofibroblast-associated markers, while E-cadherin is an epithelial cell marker. The present study therefore assessed the protein and mRNA expression of these genes in order to elucidate the effects of miR-200a on the EMT. RT-qPCR and western blot analysis indicated that overexpression of miR-200a led to a reduction of the mRNA and protein expression of the myofibroblastic markers type I collagen and $\alpha$-SMA and desmin, while the expression of epithelial cell marker E-cadherin was upregulated $(\mathrm{P}<0.05)$ (Fig. 1C and D). In analogy with these results, immunofluorescence staining further confirmed that the levels of type I collagen and $\alpha$-SMA were reduced by miR-200a overexpression, while E-cadherin expression was enhanced (Fig. 2). These results suggested that miR-200a overexpression inhibited the EMT of HSCs, indicating that miR-200a may attenuate liver fibrosis partly through inhibiting EMT.

miR-200a prevents EMT by interfering with Hh pathway activity. A previous study demonstrated that EMT is be promoted by the activation of the Hh pathway in primary rat HSCs (7). As the Hh pathway is involved in the regulation of the EMT, the present study detected the effects of miR-200a on the expression of Hhip (a competitive antagonist of $\mathrm{Hh}$ ligands), Shh (Hh ligand), Gli1 and Gli2 (Hh target genes) in primary HSCs. The results revealed that the expression of Hhip, Shh and Gli1 was not affected by miR-200a mimics (Fig. 3A and B). However, the expression of Gli2, a downstream signaling molecule of the Hh pathway, was markedly inhibited by miR-200a at the mRNA and protein level $(\mathrm{P}<0.05)$ (Fig. 3A and B). These results indicated that the Hh pathway was inhibited by miR-200a. In general, upregulation of the Hh pathway is associated with altered expression of several factors that regulate EMT, including decreases in BMP-7 and Id2 (genes that inhibit EMT) and increases in Snail and S100A4 (genes that promote EMT) (9). The present study further investigated the effects of miR-200a on genes involved in the EMT. The mRNA and protein expression of BMP-7 and Id 2 was significantly upregulated following miR-200a overexpression, while the mRNA expression of Snai1 and S100A4 was downregulated $(\mathrm{P}<0.05)$ (Fig. $3 \mathrm{C}$ and $\mathrm{D})$. These results suggested that miR-200a suppressed EMT via the Hh pathway.

Gli2 is a direct target of miR-200a in rat HSC. To confirm the underlying mechanism of miR-200a suppressing the $\mathrm{Hh}$ pathway, a bioinformatics analysis using Targetscan was performed to search for targets of miR-200a. Gli2, a downstream component of the Hh pathway, was predicted as a putative target of miR-200a. Next, the present study used a luciferase reporter assay to investigate whether Gli2 was a direct target of miR-200a. The miR-200a-specific target region of the 3'UTR of Gli2 mRNA was cloned into the pMIR-Report ${ }^{\mathrm{TM}}$ Luciferase plasmid, which was co-transfected into primary HSCs along with miR-200a precursor or miR-NC (Fig. 4A and B). $\beta$-gal reporter control plasmid was co-transfected to monitor transfection efficiency. The results showed that the luciferase activity in the reporter plasmid containing the wild-type 3'UTR of Gli2 was significantly reduced by miR-200a precursor, while the luciferase activities of the plasmid containing the mutated-type Gli2 3'UTR and empty vector were not affected (Fig. 4C). In addition, Gli2 expression was reduced by miR-200a mimics in primary HSCs (Fig. 3A and B). These results suggested that Gli2 was a direct target of miR-200a.

\section{Discussion}

Emerging studies have indicated that the EMT is involved in the transformation of Q-HSCs into MF-HSCs $(9,20)$. During the transformation of Q-HSC into MF-HSC in the present study, HSCs acquired a more mesenchymal phenotype as characterized by robust expression of several myofibroblastic markers, including $\alpha$-SMA and Col1A1. Simultaneously, the epithelial characteristics, including E-cadherin expression, were lost in the EMT process. This observation was in accordance with those of a previous study, which reported that freshly isolated primary Q-HSC expressed epithelial markers and subsequently acquired a myofibroblastic phenotype via EMT under standard culturing conditions (9). For this reason, the present study used freshly isolated primary HSCs (3 days following isolation) in all experiments.

A previous study showed that miR-200a exhibits activity against liver fibrosis (13); however, the underlying mechanism and the possible involvement of the ECM had remained elusive. Therefore, the present study assessed the mechanism of action of miR-200a in HSCs and identified its direct target. Initially, the present study revealed that miR-200a was reduced during activation of primary HSCs and in HSCs isolated from a rat model of liver fibrosis; furthermore, miR-200a upregulation and contributed to the suppression of activated HSCs, leading to a reduction in cell proliferation, ECM production and $\alpha$-SMA expression. The results of the present study were consistent with those of a previous study (13). miRNAs are known to function as key factors to regulate cell proliferation, differentiation and apoptosis (21). miRNAs are also involved in processes associated with the EMT. For example, a recent study reported that the EMT of colon cancer cells was reversed by resolving the inhibition of miR-200 cluster expression (22). However, few studies regarding the regulation of the EMT by miRNAs in liver fibrosis have been performed (23). The present 
study assessed the effects of miR-200a on the EMT in primary HSCs. Of note, myofibroblastic markers $\alpha$-SMA, Col1A1 and desmin were reduced by miR-200a in primary HSCs at the protein and mRNA level, while the expression of epithelial marker E-cadherin was increased. Immunofluorescence staining further confirmed these findings. These results suggested that miR-200a inhibited the activation of HSCs via the inhibition of the EMT.

Next, the present study investigated the molecular mechanism by which miR-200a inhibited the EMT in primary HSCs. Numerous studies have demonstrated that the Hh pathway is involved in the EMT of activated HSCs, and that it modulates MF-HSC accumulation and liver fibrosis $(9,10,24,25)$. For instance, increased expression of Shh ligand during liver injury was shown to drive EMT by promoting MF-HSC proliferation and viability (26). The Hh pathway is important in the transition of Q-HSC to MF-HSC, as Hh ligands were shown to be required for the transformation of Q-HSC into MF-HSC and to be essential for MF-HSC to retain their fibroblastic phenotype $(24,26)$. These studies suggested that activation of the $\mathrm{Hh}$ pathway may promote liver fibrosis via the EMT. Therefore, when Hh ligands interact with their receptors, the Hh pathway is activated, resulting in the activation and nuclear localization of GLI family transcription factors (27). For this reason, the present study assessed the effects of miR-200a on the activation of the Hh pathway in primary HSCs. The results showed that miR-200a inhibited the expression of the Hh downstream signaling molecule Gli2, while Hhip, Shh and Gli1 were not affected. A previous study showed that the suppression of the Hh pathway led to changes in the expression of genes associated with the EMT, including an increase of EMT inhibitors BMP-7 and Id2 and a reduction of EMT promoters Snai1 and S100A4 (28). Consistent with the results of this previous study, the present study found that miR-200a mimics induced an increase of BMP-7 and Id 2 while reducing the expression of Snail and S100A4. These results confirmed the inhibition of downstream signaling processes of the $\mathrm{Hh}$ pathway by miR-200a. To identify the target of miR-200a within the Hh pathway, a bioinformatics analysis with Targetscan was used. The downstream signaling molecule Gli2 was indicated to be a direct target of miR-200a, which was experimentally verified using a dual luciferase reporter assay. A previous study indicated the role of miR-200 family in regulating TGF- $\beta 1$-induced EMT in renal tubular cells through the Smad pathway by targeting ZEB1 and ZEB2 expression (14); however, the mechanism of action of miR-200a in liver fibrosis as well as the effects of other miR-200 family members on the EMT have remained elusive. The present study suggested that miR-200a inhibited EMT via reducing Hh signaling, at least in part, via its direct target Gli2. Targetscan analysis indicated that other members of the Hh signaling pathway, including Hhip, Gli1 and Gli2, were not targeted by any additional miR-200 family members (i.e., miR-200b, miR-429, miR-200c and miR-141) (data not shown). These results indicated that miR-200a had a unique and vital role in the Hh pathway.

In conclusion, the results of the present study provided novel insight into the role of miRs in liver fibrosis; miR-200a inhibited the fibrosis-associated EMT process in HSCs, at least in part, via blocking Gli2, a downstream signaling molecule of the Hh pathway.

\section{Acknowledgements}

The authors of the present study are grateful to Dr Zhou for the donation of specific primers containing the desired mutation. The study was supported by the National Natural Science Foundation of China (grant nos. 81000176/H0317, 81100292/ H0317, and 81500458/H0317), the Zhejiang Provincial Natural Science Foundation of China (grant nos. Y2090326, Y2110634 and LY16H030012), the Wang Bao-En Liver Fibrosis Foundation (grant nos. 20100002 and 20120127) and the Wenzhou Municipal Science and Technology Bureau (grant nos. Y20110033 and Y20120127).

\section{References}

1. Yue HY, Yin C, Hou JL, Zeng X, Chen YX, Zhong W, Hu PF, Deng X, Tan YX, Zhang JP, et al: Hepatocyte nuclear factor 4alpha attenuates hepatic fibrosis in rats. Gut 59: 236-246, 2010.

2. Friedman SL: Hepatic stellate cells: Protean, multifunctional, and enigmatic cells of the liver. Physiol Rev 88: 125-172, 2008.

3. Ogawa T, Iizuka M, Sekiya Y, Yoshizato K, Ikeda K and Kawada N: Suppression of type I collagen production by microRNA-29b in cultured human stellate cells. Biochem Biophys Res Commun 391: 316-321, 2010.

4. Kawada N: The hepatic perisinusoidal stellate cell. Histol Histopathol 12: 1069-1080, 1997.

5. Lee JM, Dedhar S, Kalluri R and Thompson EW: The epithelial-mesenchymal transition: New insights in signaling, development, and disease. J Cell Biol 172: 973-981, 2006.

6. Hernandez-Gea V and Friedman SL: Pathogenesis of liver fibrosis. Ann Rev Pathol 6: 425-456, 2011.

7. Omenetti A, Porrello A, Jung Y, Yang L, Popov Y, Choi SS, Witek RP, Alpini G, Venter J, Vandongen HM, et al: Hedgehog signaling regulates epithelial-mesenchymal transition during biliary fibrosis in rodents and humans. J Clin Invest 118: 3331-3342, 2008.

8. Zeisberg M, Yang C, Martino M, Duncan MB, Rieder F, Tanjore H and Kalluri R: Fibroblasts derive from hepatocytes in liver fibrosis via epithelial to mesenchymal transition. J Biol Chem 282: 23337-23347, 2007.

9. Choi SS, Omenetti A, Witek RP, Moylan CA, Syn WK, Jung Y, Yang L, Sudan DL, Sicklick JK, Michelotti GA, et al: Hedgehog pathway activation and epithelial-to-mesenchymal transitions during myofibroblastic transformation of rat hepatic cells in culture and cirrhosis. Am J Physiol Gastrointest Liver Physiol 297: G1093-G1106, 2009.

10. Choi SS, Syn WK, Karaca GF, Omenetti A, Moylan CA, Witek RP, Agboola KM, Jung Y, Michelotti GA and Diehl AM: Leptin promotes the myofibroblastic phenotype in hepatic stellate cells by activating the hedgehog pathway. J Biol Chem 285: 36551-36560, 2010.

11. Bartel DP: MicroRNAs: Genomics, biogenesis, mechanism, and function. Cell 116: 281-297, 2004.

12. Croce $\mathrm{CM}$ and Calin GA: miRNAs, cancer, and stem cell division. Cell 122: 6-7, 2005.

13. Sun X, He Y, Ma TT, Huang C, Zhang L and Li J: Participation of miR-200a in TGF- $\beta 1$-mediated hepatic stellate cell activation. Mol Cell Biochem 388: 11-23, 2014.

14. Xiong M, Jiang L, Zhou Y, Qiu W, Fang L, Tan R, Wen P and Yang J: The miR-200 family regulates TGF- $\beta 1$-induced renal tubular epithelial to mesenchymal transition through Smad pathway by targeting ZEB1 and ZEB2 expression. Am J Physiol Renal Physiol 302: F369-F379, 2012.

15. Weiskirchen R and Gressner AM: Isolation and culture of hepatic stellate cells. Methods Mol Med 117: 99-113, 2005.

16. Yao QY, Xu BL, Wang JY, Liu HC, Zhang SC and Tu CT: Inhibition by curcumin of multiple sites of the transforming growth factor-betal signalling pathway ameliorates the progression of liver fibrosis induced by carbon tetrachloride in rats. BMC Complement Altern Med 12: 156, 2012.

17. Zheng J, Wu C, Lin Z, Guo Y, Shi L, Dong P, Lu Z, Gao S, Liao Y, Chen B and Yu F: Curcumin up-regulates phosphatase and tensin homologue deleted on chromosome 10 through microRNA-mediated control of DNA methylation-a novel mechanism suppressing liver fibrosis. FEBS J 281: 88-103, 2014. 
18. Schmittgen TD and Livak KJ: Analyzing real-time PCR data by the comparative C(T) method. Nat Protoc 3: 1101-1108, 2008.

19. Xing CY, Hu XQ, Xie FY, Yu ZJ, Li HY, Bin Z, Wu JB, Tang LY and Gao SM: Long non-coding RNA HOTAIR modulates c-KIT expression through sponging miR-193a in acute myeloid leukemia. FEBS Lett 589: 1981-1987, 2015.

20. Choi SS, Witek RP, Yang L, Omenetti A, Syn WK, Moylan CA, Jung Y, Karaca GF, Teaberry VS, Pereira TA, et al: Activation of Racl promotes hedgehog-mediated acquisition of the myofibroblastic phenotype in rat and human hepatic stellate cells. Hepatology 52: 278-290, 2010.

21. Schickel R, Boyerinas B, Park SM and Peter ME: MicroRNAs: Key players in the immune system, differentiation, tumorigenesis and cell death. Oncogene 27: 5959-5974, 2008.

22. Tian Y, Pan Q, Shang Y, Zhu R, Ye J, Liu Y, Zhong X, Li S, He Y, Chen L, et al: MicroRNA-200 (miR-200) cluster regulation by achaete scute-like 2 (Ascl2): Impact on the epithelial-mesenchymal transition in colon cancer cells. J Biol Chem 289: 36101-36115, 2014.
23. Csak T, Bala S, Lippai D, Kodys K, Catalano D, Iracheta-Vellve A and Szabo G: MicroRNA-155 deficiency attenuates liver steatosis and fibrosis without reducing inflammation in a mouse model of steatohepatitis. PLoS One 10: e0129251, 2015.

24. Yang L, Wang Y, Mao H, Fleig S, Omenetti A, Brown KD, Sicklick JK, Li YX and Diehl AM: Sonic hedgehog is an autocrine viability factor for myofibroblastic hepatic stellate cells. J Hepatol 48: 98-106, 2008.

25. Jung Y, Brown KD, Witek RP, Omenetti A, Yang L, Vandongen M, Milton RJ, Hines IN, Rippe RA, Spahr L, et al: Accumulation of hedgehog-responsive progenitors parallels alcoholic liver disease severity in mice and humans. Gastroenterology 134: 1532-1543, 2008.

26. Jung Y, Witek RP, Syn WK, Choi SS, Omenetti A, Premont R, Guy CD and Diehl AM: Signals from dying hepatocytes trigger growth of liver progenitors. Gut 59: 655-665, 2010.

27. Kalderon D: Transducing the hedgehog signal. Cell 103: 371-374, 2000.

28. Zeisberg $\mathrm{M}$ and Neilson EG: Biomarkers for epithelial-mesenchymal transitions. J Clin Invest 119: 1429-1437, 2009. 